\title{
Improving Brain MR Image Classification for Tumor Segmentation using Phase Congruency
}

\author{
Ghazanfar Latif $^{*}, 1,2$, D.N.F. Awang Iskandar ${ }^{1}$, Jaafar Alghazo ${ }^{2}$ and Arfan Jaffar ${ }^{3}$ \\ ${ }^{I}$ Faculty of Computer Science and Information Technology, Universiti Malaysia Sarawak, Malaysia; ${ }^{2}$ College of Com- \\ puter Engineering and Sciences, Prince Mohammad bin Fahd University, Saudi Arabia; ${ }^{3}$ Department of Computer Sci- \\ ence, Al-Imam Muhammad Ibn Saud Islamic University, Saudi Arabia
}

\begin{abstract}
Background: MRI which stands for Magnetic Resonance Imaging is commonly used to capture images of internal body organs, functionality and structure. Manual analysis is usually performed by Radiologists on a large set of MR images in order to detect brain tumor.
\end{abstract}

Aims: This research aims to improve automated brain MR image classification and tumor segmentation using phase congruency.

\author{
A R T I C L E H I S T O R Y \\ Received: January 22, 2018 \\ Revised: March 06, 2018 \\ Accepted: March 19, 2018 \\ DOI \\ $10.2174 / 1573405614666180402150218$
}

\begin{abstract}
Methods: The skull part is removed from brain MR image by applying converging square algorithm and phase congruency based edge detection method. Features are then extracted from the segmented brain portion using discrete wavelet transforms. In order to minimize the extracted feature set, we applied the principal Component Analysis algorithm. The MR images are classified into tumorous and non-tumorous using Multilayer perceptron and compared with other classifiers such as K-Nearest Neighbor, Naïve Bayes, and Support Vector Machines (SVM) along with discrete cosine and discrete cosine transform features. The tumor is segmented using Fuzzy C-mean and reconstructed tumor $3 \mathrm{D}$ model to measure the volume, location and shape accurately.
\end{abstract}

Results \& conclusions: Experimental results are obtained by testing the proposed method on a dataset of 19 patients with a total number of 2920 brain MR images. The proposed method achieved an accuracy of $99.43 \%$ for classification which is higher as compared to other current studies.

Keywords: Brain MRI, phase congruency, segmentation, tumor analysis, feature extraction, tumor classification.

\section{INTRODUCTION}

An abnormal growth of brain cells known as brain tumor is the main cause of death in patients [1]. In 2008, $13 \%$ of the 7.6 million deaths worldwide were attributed to cancer [2]. These figures were obtained from the International Agency for Research on Cancers report which was published in 2013 and indicates that the death toll due to cancer is increasing annually [2]. The World Health Organization report states that by 2030 there will be an estimated 22.2 million people with cancer [3]. This will affect mostly developing countries such as Kenya, Ghana, and Afghanistan because the statistics show that almost $70 \%$ of deaths worldwide occur in developing countries.

Though brain tumor can be classified into many different types, yet medical doctors usually classify them into either benign or malignant. Tumor classified as benign are noncancerous while those classified as malignant may spread to neighboring tissue or other portions of the brain and may lead to brain cancer. Magnetic Resonance Images (MRI) is a

*Address correspondence to this author at the Faculty of Computer Science and Information Technology, Universiti Malaysia Sarawak, 94300 Kota Samarahan, Sarawak, Malaysia; E-mail: glatif@pmu.edu.sa technologically advanced medical imaging method used for the diagnosis and interpretation of abnormalities in the human body. In particular, brain MRI is advanced to the extent that it goes through a series of segmentation and detection to identify normal and cancerous tissues. On average, Brain MRI produces about 150-300 detailed images for each patient.

It takes doctors anywhere from 30-45 minutes to properly interpret brain MRI scans. This requires very experienced radiologists which are scarce in developing countries [4]. The complexity of diagnosing brain tumor lies in the difficulty of segmenting the overlapping tissues from different brain structures or other non-brain tissue. This may lead in some cases to misdiagnosis of brain tumor.

The process of segmentation has gained importance in the medical field especially in brain MRI segmentation, breast MRI segmentation and lung CT-Scan image segmentation among others. [5]. Three types of tissue exist in the human brain namely; cerebrospinal fluid, grey matter and white matter [6]. Due to the similarity in intensity between these tissue types, it becomes difficult to segment one type from another. Thus, the main contribution of this research is on the segmentation stage of brain images and then classifying the segmented image into tumorous or non-tumorous. 